\title{
Lung artefacts and their use
}

\author{
Christoph F Dietrich ${ }^{1,2}$, Gebhard Mathis ${ }^{3}$, Michael Blaivas ${ }^{4}$, Giovanni Volpicelli ${ }^{5}$, \\ Armin Seibel ${ }^{6}$, Nathan S Atkinson ${ }^{7}$, Xin Wu Cui ${ }^{1,8}$, Fan Mei ${ }^{1}$, Dagmar Schreiber-Dietrich ${ }^{2}$, \\ Dong $\mathbf{Y i}^{9}$
}

\begin{abstract}
${ }^{1}$ Sino-German Research Center of Ultrasound in Medicine, The First Affiliated Hospital of Zhengzhou University, Zhengzhou, China, ${ }^{2}$ Department of Internal Medicine, Caritas Hospital, Bad Mergentheim, Germany ${ }^{3}$ Praxis for Internal Medicine, Rankweil, Austria, ${ }^{4}$ University of South Carolina School of Medicine, Department of Emergency Medicine, Piedmont Hospital, Newnan Georgia, USA, ${ }^{5}$ Department of Emergency Medicine, San Luigi Gonzaga University Hospital, Torino, Italy, ${ }^{6}$ Diakonie Klinikum Jung-Stilling, Abteilung für Anästhesiologie, Intensiv- und Notfallmedizin, Siegen, Germany, ${ }^{7}$ Department of Gastroenterology, John Radcliffe Hospital, Oxford University Hospitals NHS Trust, ${ }^{8}$ Department of Medical Ultrasound, Tongji Hospital, Tongji Medical College, Huazhong University of Science and Technology, Wuhan, China, ${ }^{9}$ Department of Ultrasound, Zhongshan Hospital, Fudan University, Shanghai, China
\end{abstract}

\begin{abstract}
The science of lung ultrasound has grown tremendously over the last two decades and lung ultrasound has not only entered the mainstream of point of care ultrasound but has become a dominant topic. Understanding lung ultrasound signs and artifacts is critical to being able to correlate findings with actual pathology and normal anatomy and physiology. Investigators have described multiple lung ultrasound artifacts and findings and it is important to understand both the physics and anatomic basis behind them. Additionally, ultrasound machine use and transducer selection can significantly affect results obtained on patient during an examination and the provider must carefully choose the correct settings. This manuscript describes the state of the art in ultrasound artifact recognition and correlation as well as management of ultrasound technology to optimize diagnostic success.
\end{abstract}

Keywords: ultrasound, lung, artifacts, pathology, guidelines

\section{Introduction}

In years gone by examination of patients with pulmonary disorders rested essentially on a clinician's bedside physical examination, of auscultation and percussion, complimented with blood gas analysis and X-ray imaging. Lung ultrasound has emerged into this context as a real-time bedside procedure, delivering information relevant to the clinician's differential diagnosis. A large part of sonographic lung examination involves the interpretation of artefacts, thus knowledge of the origin of typi-

Received Accepted

Med Ultrason

2016, Vol. 18, No 4, 488-499

Corresponding author: Prof. Dr. Christoph F. Dietrich

Med. Klinik 2

Caritas-Krankenhaus Bad Mergentheim

Uhlandstr. 7

97980 Bad Mergentheim, Germany

E-mail: Christoph.dietrich@ckbm.de cal artefacts is important. Although indirect sonographic signs, nonetheless reliable conclusions on the condition of the lung can be inferred. Particularly the of quantity and distribution of B-lines can establish important assessment of the cause and degree of fluid load within the interstitium.

All focused ultrasound investigations, including bedside lung ultrasound, allow symptom evaluation considering multiple organ-systems yet with minimal time delay. Such integration of imaging with clinical assessment and treatment is called Point-of-Care Ultrasound (POCUS) and should be understood as an extension of the physical examination. POCUS offers a unique but crucial role, integrating clinical and other imaging findings, including cardiac ultrasound (echocardiography), chest radiography, and computed tomography (CT) scans is crucial, which alone may lack the required accuracy.

The emergence of differences in approach to lung sonography, technique and nomenclature, provided the 
incentive for a consensus process examining six major areas; terminology, technology, technique, clinical outcomes, cost effectiveness and future research. A scientific pathway process was followed, to generate evidencebased guidelines with recommendations for clinical lung ultrasound applications [1]. B-line artefacts and their use have been recently described in detail [2].

In this review in addition to a series of papers about point of care ultrasound $[3,4]$, lung ultrasonography $[5,6]$, mediastinal ultrasound [7-10] and artefacts [2,11-15] we discuss the prominent role of the particular artefacts of lung sonography answering differential-diagnostic questions in patients with cardio-pulmonary diseases.

\section{Lung artefacts}

\section{Introduction into artefacts \\ B-mode ultrasound (grey scale) \\ Definition}

Artefacts are unintentionally generated phenomena, a discrepancy between assumptions and reality due to method-based and system-inherent image errors. Causes of image errors may relate to equipment defects requiring repair of the technology, or interference from within or outside, including those caused by the examiner or other influencing factors. Real artefacts must be understood to be interpreted correctly. Reduced depth penetration or low resolution in the near filed may be improved by changes of the transducer frequency, persistence and other maneuvers. Attenuation of sound propagation is per se also an artefact as it can be compensed for by depth gain adjustment $[11,16]$.

Primarily artefacts should be named according to their underlying mechanism (e.g., reverberation caused by mirroring) and secondarily according to the imaging features (appearance), e.g., comet-tail artifact. Recent consensus recommendations termed the comet-tail artifact, caused by reverberation at the pleura line, as the ' $\mathrm{B}$ line artefact'. Alternative terms should be avoided to reduce confusion.

\section{Assumptions}

The pulse-echo principles and assumptions made by ultrasound machines explain artifact formation. Important physical principles include "differences in impedance" on the surface from one anatomical structure to another, result in differences of echo amplitude and intensity (strength). Put simply, the transmitted pulse generates reflections and scatter at acoustic boundary surfaces. Several important assumptions occur within the ultrasound machine processing including; a straight line of a constant sound propagation speed of $1540 \mathrm{~m} / \mathrm{s}$ without any interactions or deviation in both directions, a val- id assumption only before and after reflection; that image reconstruction time is continuous and negligible (without any interruptions); that sound attenuation is equal at every image depth over the entire ultrasound image; that a one-dimensional sound lobe line with a defined and limited length has no other dimension (no thickness or width); and that echo amplitude and intensity (strength) depend only upon the anatomical structures from which they are reflected (back scatter). Artefacts caused by the assumptions and discrepancies of the true dimensions of sound lobes are called slice-thickness artefacts, e.g., in the case of width bow artefacts $[11,16]$.

\section{Mirror artefacts}

Mirror artefacts are particularly important in lung ultrasound and can be explained by the physical principles of mirroring of light (highly reflective, large objects in relation to the wavelength). They vary according to changes of the shape, surface and sound semi-permeability of the mirror, the angle between the sound beam and mirror surface, and occur as axial (A- and B-lines) and non-axial mirroring (according to the rules of geometry). Axial mirroring is caused by pulse-echo reverberations multiply reflected back and forth between two reflective surfaces. Reflections can occur between two reflective surfaces, or one surface and the transducer. Axial mirroring can be identified and reduced by changing the insonation angle or compression with the transducer. Real anatomical structures shift in the same direction whereas axial mirror artefacts shift in the opposite direction. Compound and harmonic imaging reduces such artefacts. The respectively rough and smooth surfaces using M-mode explain the differences of the normal lung (so-called seashore sign) and pneumothorax with the socalled barcode-sign (if there are no pathological changes of the pleural line). In the case of very small and strong echogenic structures (e.g., air, metal, calcifications or cholesterol particles) reverberation results in comet-tail or ring down artefacts depending on the size of the particle [17]. Ring down artefacts are explained by resonance ("vibration" with signal extension) due to fluid between gas bubbles, metal and other structures $[11,16]$.

\section{Range ambiguities}

So-called range ambiguities occur when the pulseechoes travel beyond the set depth penetration with reflection of resulting echoes too close to the transducer.

\section{Color Doppler imaging}

\section{Definition}

Color Doppler imaging allows display of the (averaged) relative velocity (variance) and direction of blood flow ("color Doppler velocity"). The Doppler signal strength of the blood stream can be displayed using so-called "power Doppler" or "color Doppler energy" [11,16]. 
Assumptions

Doppler signals are generated only by the contents of the blood vessels (and not by tissue structures).

\section{Lung artefacts}

According to international consensus, relatively few lung artefacts are important for the diagnosis of (interstitial) lung diseases [1]. The recognition of B-line artefacts (BLA) gives additional information, for example excluding the presence of a pneumothorax.

\section{Pathophysiology}

The pathophysiological changes within the interstitial tissues necessary to generate diffuse BLA with ultrasound have been identified by CT studies as abnormalities of the secondary pulmonary lobule including: thickening of the interlobular septa, areas of ground-glass opacity, and diffuse alveolar opacities $[18,19]$. These abnormalities are seen in a variety of interstitial lung conditions, characterized by the presence of interstitial fluid, cellular infiltration and fibrosis. Experts grouped the diffuse pulmonary conditions that may produce similar BLA (multiple, diffuse and bilateral BLA on ultrasound) into three main categories: extravascular lung water (EVLW) due to increased hydrostatic pressure or increased capillary bed permeability, infections causing interstitial inflammation or fibrosis, and infiltrative processes of the interstitium [1].

Soldati et al [20] considered the 'spongy' nature of the lung in its liquid and solid components and the changes that take place in peripheral airspace geometry, which might be the key to understanding these phenomena. From animal experiments, they concluded that airspace geometry, frothy nature and porosity are the determinants of the different behaviour of ultrasound wave interactions with the sub pleural lung parenchyma. Chest ultrasound may be interpreted as an indirect 'estimator' of lung porosity [20].

"Ring down" artefacts, appear as a solid streak of parallel bands radiating away from air bubbles and so differ in pathophysiological mechanism from "comet tail" artefacts. However, ring down artefacts rarely present a problem of differentiation with BLA, which are only significant when multiple, close to each other and repetitive, whereas the ring down artefacts are usually isolated or few in number. Visualization of isolated BLA, or multiple BLA separated by more than $7 \mathrm{~mm}$ in a single scan, as well as the appearance of ring down artefacts, is considered a normal finding [1].

\section{A-line artefact (ALA)}

A-line artefacts (ALA) are defined as horizontal linear artefacts in the lung image, repeating the same chest wall to pleural line distance. This reverberation phenomenon is due to a mirror effect generated by a normal (or

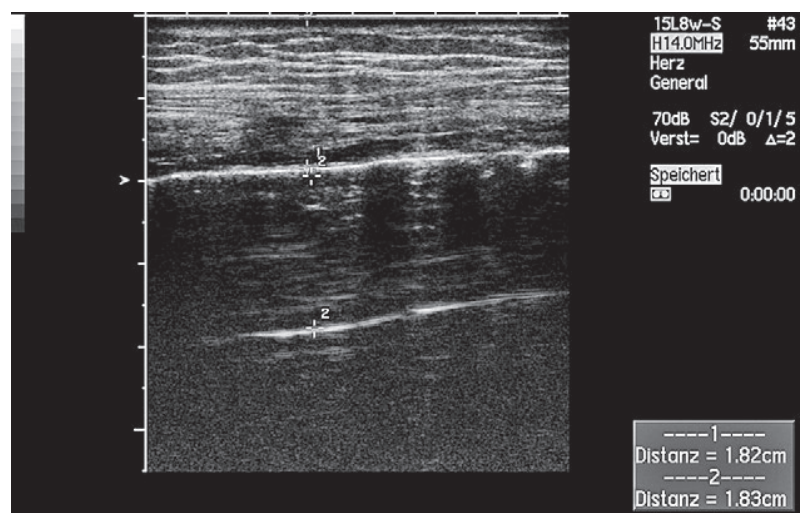

Fig 1. A-line artefact (ALA) is shown as horizontal repetitions of the pleural line.

enhanced) balance of subpleural air, which blocks the propagation of the ultrasound beam. A pattern showing a normal lung surface, typically associated to lung sliding, with horizontal repetitions of the pleural line is called Apattern (fig 1). Lung sliding is a to-and-fro movement at the pleural line occurring with ventilation. ALA without sliding may indicate free air in the pleural space. Correlation with other signs is necessary to conclude a pneumothorax is present [21].

Other lung artefacts

Other lung artefacts have been described but they have much less use for lung ultrasound and should be avoided [1]. E-lines are a sign of subcutaneous emphysema (E for emphysema) and are long vertical artefacts similar distinguishable from BLA, as they do not arise from the pleural line [22]. Finally, alveolar consolidations are shown as the presence of anechoic or tissue-like subpleural structures [23].

\section{Examining lung artefacts}

The ultrasound examination consists of bilateral scanning of the anterior and lateral chest walls performed on patients in a supine or near to supine position, which is often mandatory in the emergency setting. This standardized position has the advantage of being applicable in any condition [24]. Each hemithorax should be examined from apex to base in three well defined regions: anterior infraclavicular, midlateral on the midaxillary line, and posterobasal subscapular regions [21] (fig 2). The consensus process defined the basic eight region sonographic technique and the criteria for a positive scan and positive examination. In the critically ill, a more rapid anterior two-region scan may be sufficient to rule out the interstitial syndrome in cardiogenic acute pulmonary edema. In acutely decompensated heart failure, semi-quantification of the severity of congestion can be calculated by counting the total number of BLA (28 scanning site technique) or the number of positive scans (eight region technique) [1]. 
BLA are best visualized during a real time examination, because some are less pronounced on frozen sonograms. A positive region is defined by the presence of three or more BLA in a longitudinal (intercostal) plane. A positive exam is defined by the presence of two or more positive regions bilaterally when the examination is performed on 4 anterolateral chest areas per side. BLA can be used to diagnose, semiquantify severity and exclude diseases.

Occasionally and depending on the specific setting and patient condition, other direct ultrasound findings should be examined (e.g., thoracic wall, pleura, pleural effusion, subpleural consolidation, diaphragm function) with the best technique available, focussing on the best detail resolution. The detailed anatomy of the diaphragm is shown in a patient with a pleural effusion.

\section{Healthy subjects}

Chiesa et al compared US appearances of the lungs in a group of healthy, non-smoking elderly subjects with those of young subjects. BLA were found in $37 \%$ of elderly subjects, but only $10 \%$ of young healthy subjects [25]. In a recently performed pilot study, we identified distinctive influencing factors on the detection and characterisation of BLA. The presence of BLA, in most cases $<3$ per field of view, is a normal finding especially in the elderly population. The reduction of impedance between lung parenchyma and soft tissues of the chest wall and the increased thickness of interlobular septa might explain these findings [25]. In conclusion, the normal lung is characterized by the absence, or presence of very few, BLA (less than three per field of view).

\section{Pathological findings}

Three or more BLA between two ribs in a single scan indicates a subpleural component of the interstitial syndrome. Semiquantification of BLA directly correlates with the amount of interstitial fluid in selected patients with pulmonary congestion. Scores to assess the severity of BLA have been adopted as follows: $0=$ none; $1=$ physiologic; $2=$ mild; $3=$ moderate; and $4=$ severe. In practice, a score of either 3 or 4 tends to increase the likelihood of a diagnosis of increased interstitial fluid, whereas a score of either 0 or 1 will decrease it [26]. For follow up examinations, it is necessary to use the same or similar technical equipment when counting BLA for the semiquantitative assessment of pulmonary diseases.

\section{Education and training}

Brattain et al developed algorithms to evaluate the feasibility of diagnostic assistants to quantify BLA in a sample set of clips from one machine. Both the left and right lung fields were scanned at a depth of $18 \mathrm{~cm}$, with each lung field visualized in 4 thoracic zones (anteriorsuperior, anterior or inferior, lateral-superior, and lateral-

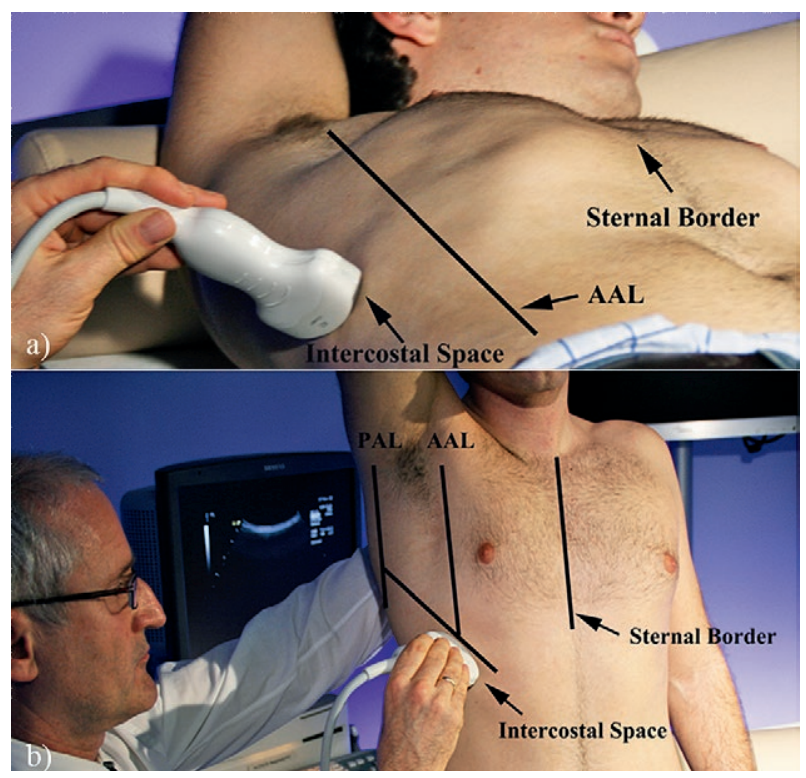

Fig 2. Examination technique. Anterior axillary line (AAL), posterior axillary line (PAL)), intercostal space, and sternal border are indicated in a supine (a) and standing (b) position in a healthy subject.

inferior). The algorithm addressed individual, operator, and BLA appearance variation due to particular statistical realizations of the closely spaced interfaces in lung. On the set used for algorithm development, $90 \%$ of the scores matched the average expert scores with differences of 1 or fewer BLA. The best match was achieved in computerized BLA scoring. The use of a diagnostic assistant provides value by providing: 1) an objective measurement that can aid subjective judgments, 2) an increase in the confidence of novice users to act on their findings, and 3) a training and feedback mechanism that could help maintain and build a novice user's diagnostic skills during training [27].

\section{Review of the literature \\ Ultrasound equipment}

Since BLA are artefacts caused by physiologic changes in the lung parenchyma, they are potentially influenced by machine settings and signal processing. Sophisticated pre- and post- processing should be turned off if possible or limited to a minimum to allow comparability.

Cogliati et al evaluated the accuracy of lung ultrasound in the diagnosis of interstitial lung disease (ILD) and validated the use of a pocket-size US device (PSUSD) as a screening tool. Their results showed good correlation between the PS-USD and standard ultrasound equipment, even when using different probes (convex versus cardiac), and good reproducibility compared with standard US equipment [28]. 
Transducer

Sperandeo et al compared the BLA examinations with low-medium frequency (3.5-5.0 MHz) convex probe and high-frequency $(8-12.5 \mathrm{MHz})$ linear probe. Counts of BLA were higher when convex probes were used [29]. However, other more accepted studies performed with convex probe [24], linear probe [30], cardiac probe [31] and microconvex probe [32] showed similar findings on the visualization of BLA in a variety of settings and patients and by using different machines. Microconvex 2-5 $\mathrm{MHz}$ transducer was recommended by Lichtenstein et al because this transducer provides an extended view of the pleural surface and penetrated deeply enough to verify the characteristic of vertical artefacts. The abdominal probe at 3-5 MHz has the advantage of coupling a wider field of view of the pleura and detection of deep structures [24].

Depth of penetration

The higher the frequency the lower the penetration but other factors also influence the depth of penetration, e.g., harmonic imaging. BLA look different at different levels, depending on the frequency and transducer shape used. The depth of penetration should be standardised to $4-8 \mathrm{~cm}$ starting from the pleura line (depending on the frequency used).

Focus

The focus of the image should be set at the level of the pleural line, focusing the most energy for reflection and reverberating. The use of multifoci is discouraged since less relative energy is encountered at the pleural line.

Preprocessing and postprocessing

Tissue harmonic imaging, compound imaging, different pre- and post-processing techniques, filters and interpolation algorithms can alter the appearance of BLA.

Comment

Indeed, there is the possibility that visualization of BLA may vary by changing technical adjustments, machines and probes. However, there are two points to consider regarding this hypothesis. The first is that the normal adjustment of the lung image should be optimized simply by regulating the gain and all the other basic settings. Image optimization is often intuitively used in general ultrasound, which is true also for lung ultrasound. In the aerated lung, the pleural line should be well visualized. The focus should be set at the level of the pleural line and the subpleural zone adjusted for optimal imaging showing the typical granular echoic aspect, regardless of the probe and frequency used. Many manufacturers offer features for consistent and reproducible image quality and reduced operator strain. The Tissue equalization modus was used in our pilot study and provides single-button optimization for 2D imaging. However, the validity of these automated programs on the optimization of lung imaging, should be further investigated and discussed.
The second consideration is that a slight variability in the number of visible BLA may have no influence on the final diagnostic adjudication. Indeed, the diagnostic cutoffs set in BLA visualization are based on a rough count of a minimum number of artefacts in a single scan and the number and distribution of positive scans. Thus, slight changes in the single visualization of BLA may rarely affect the final diagnosis. This is also true for more sophisticated monitoring techniques, such as the one used for monitoring pulmonary congestion, based on a rough count of the percentage of the scan occupied by BLA [33]. However, new studies are needed to verify the exact influence of diverse adjustments, machines, probes and frequencies on the image generation of BLA, focalized on whether these changes may effectively bias the diagnostic criteria in lung ultrasound. In the specific pneumology setting, such as the high resolution imaging study of pulmonary fibrosis, these influencing factors may be of more importance than in the emergency setting.

\section{Are BLA reproducible?}

Adults

Agricola et al obtained intra- and inter-observer variability of $3.1 \%$ and $4.4 \%$, on 280 scans performed by two operators [31]. Boussuges et al obtained interobserver variability $<5 \%$, on 1890 scans performed by two operators [34]. Fagenholz et al. obtained "excellent agreement" on 308 scans [35]. Jambrik et al obtained intra- and interobserver variability of $5.1 \%$ and $7.4 \%$, on 560 scans performed by two operators [36]. Liteplo et al obtained an interobserver reliability Cohen kappa index of 0.82 on 800 scans performed by several operators with different skill levels and expertise [37]. Mallamaci et al obtained an inter-observer concordance index of 0.96 on 672 scans [38]. Volpicelli et al obtained inter-observer variability of $4.9 \%$ on 192 scans performed by 5 operators [24]. In a recently published large multicenter study, Pivetta et al obtained a Kappa statistic for agreement of 0.94 on 1200 scans performed by several operators, reviewed by an expert and two residents with limited training [39]. In the same study, intraobserver agreement was 0.97 for the expert operator and 0.92 for the physicians with limited training.

Anderson et al demonstrated that there is substantial or near perfect interobserver reliability among emergency sonologists using 3 different definitions for quantifying BLA in a single intercostal space. Among these methods, using percentages of rib spaces covered by fused BLA and counting the BLA at the instant of highest incidence, result in higher reliability [40]. Sperandeo et al investigated the clinical applicability of transthoracic lung ultrasound (TLUS) in the diagnosis and follow up of community-acquired pneumonia (CAP). Concerning the reproducibility of TLUS method, no reader's bias was present. Overall 
variability and between subject variability (inter-reader agreement) did not show any difference between readers and estimated within-subject variability (intra-reader agreement) suggested a high repeatability of the method [41]. Gullett et al concluded that interobserver agreement was best in the anterior/superior thoracic zones followed by the lateral/superior zones for both expert/expert and expert/novice pairs. Agreement in the lateral/inferior lung zones was overall inferior. Intrarater agreement was highest at extreme high or low numbers of BLA [42].

BLA are highly reproducible and identifying them is easy to learn by operators with different skill and expertise.

\section{Geriatric patients}

The senile lung is characterized by mild changes on ultrasound and CT. Chest areas positive for BLA increase with age and focally multiple BLA can be found. However diffuse patterns, especially in symptomatic subjects, suggest a different diagnosis [43].

\section{Children}

Lung ultrasound is non-ionizing, which is especially important in infants who carry a higher risk of cancer from exposure to radiation than older patients [44]. Martelius et al compared the amount of BLA on sonography to the extent of parenchymal changes on computed tomography (CT) in children. They concluded that the number of BLA on sonography correlates with the extent of parenchymal changes on CT. Various parenchymal changes were seen in patients with BLA. BLA were more frequently seen in patients with no changes on $\mathrm{CT}$ when imaged during general anaesthesia [45].

\section{The use of BLA in clinical practice}

As mentioned above, it is crucial to know the current complaints, clinical condition, medical history, physical examination and imaging findings to interpret ultrasound findings of the thorax and lung. In other words, does the severity of the patient's clinical condition correlate with the extension and diffuse pattern of BLA? This question should always be answered before taking bedside decisions based on lung ultrasound BLA findings [46]. The meaning of BLA, which is by definition a sign with low specificity, may dramatically change.

Which diseases can be differentiated using BLA?

BLA diagnoses a loss of peripheral lung aeration due to interstitial disease involvement, but considering only BLA it is not possible to differentiate the cause. BLA appear with pulmonary edema (diffusely and homogeneously distributed), lung contusions, pneumonia and acute respiratory distress syndrome (ARDS) most commonly (typically focally or inhomogeneously distributed). If ARDS is diffuse, it can appear like pulmonary edema and potentially so too can bilateral lung contusions. While they have been reported in pulmonary edema, pulmonary fibrosis, pneumonia, or acute respiratory distress syndrome, single focal BLA can also be observed in healthy individuals [47]. However when integrated with clinical decision making, one can differentiate pulmonary edema, ARDS, interstitial pneumonia, pulmonary fibrosis, lung contusions and others.

The presence of multiple diffuse bilateral BLA indicates the interstitial syndrome and the number of lines increases with decreasing air content and increasing lung density. Causes of the interstitial syndrome include [1]: pulmonary edema of various causes (including cardiogenic pulmonary edema and ARDS), interstitial pneumonia or pneumonitis, and diffuse parenchymal lung disease (pulmonary fibrosis).

Lung artefacts and BLA in congestive heart failure

Facchini et al evaluated patients with heart failure for the number of BLA at TLUS, along with other noninvasive methods for identification of pulmo nary congestion (fig 3). Their results showed thoracic fluid content (TFC) value $\geq 35 / \mathrm{k} \Omega$ and BLA $\geq 15$ had the best sensitivity $(87 \%)$ and specificity (93\%) for its detection. Using at least two indices improved sensitivity and specificity [47]. BLA significantly correlated with more established parameters of chronic heart failure. BLA and their changes after therapy correlated with chest X-ray water score. A Bline cutoff $\geq 15$ provided a quick and reliable assessment of decompensation in outpatients with significant pulmonary congestion (sensitivity $85 \%$, specificity $83 \%$ ) [48].

Dyspnea is the most common and distressing symptom of patients with heart failure. Fifty-eight outpatients with moderate to severe heart failure were prospectively studied using a 5-point Likert scale for dyspnea evaluation in comparison to the degree of pulmonary congestion. They determined the prognostic value of this scale for predicting adverse events in heart failure outpatients. TLUS with $\geq 15$ BLA were used as reference for pulmonary congestion. A higher degree of dyspnea ( 3 or 4 points on the 5-point Likert scale) significantly correlated with a higher number of BLA. A symptom scale

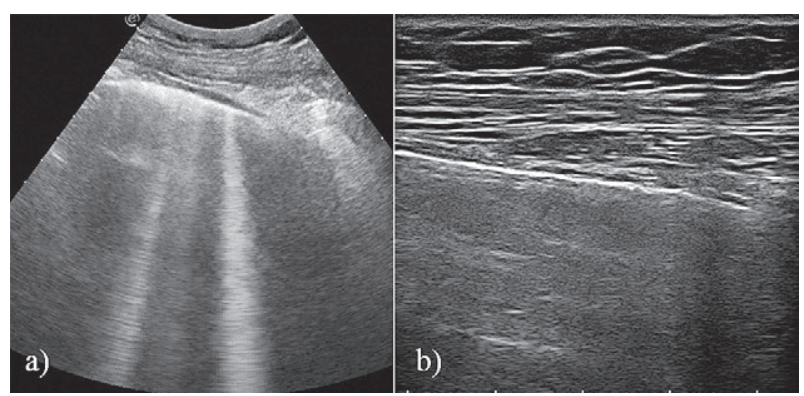

Fig 3. Chronic heart failure NYHA III with dyspnea and chronic obstructive pulmonary disease. B lines are shown using 5 $\mathrm{MHz}$ but not using $10 \mathrm{MHz}$. 
with BLA assessment could help distinguish patients in the same functional class and predict clinical outcomes during follow up [49].

Exercise induced pulmonary edema during deep apnoea diving at sea level could be evaluated using LUS and BLA [50]. High intensity intermittent exercise led to an increase in BLA at altitude after subacute exposure but not during acute exposure at equivalent simulated altitude. This may indicate pulmonary interstitial edema due to prolonged exposure to exercise at altitude. This phenomenon seems to be dependent to the length of exposure and exercise [51].

In the acute presentation critically ill patient, absence of BLA correlates better with low extravascular lung water than low wedge pressure [52]. This phenomenon indicates that the strength of BLA evaluation to provide insight into the pulmonary interstitium and to differentiate a condition of pure hemodynamic congestion from pulmonary interstitial edema.

Some studies indicate good correlation between the number of BLA and the grade of pulmonary edema. In patients admitted with acute dyspnoea, a semi-quantification of BLA correlated significantly with N-terminal B-type natriuretic peptide (NT-proBNP) values. The accuracy of BLA in predicting the cardiac origin of dyspnoea is high $[24,35,53]$. Increased extravascular lung water (EVLW) is the main determinant of multiple and diffuse BLA on chest ultrasonography. BLA changed with exposure to high altitude in a parabolic-like pattern within the first $72 \mathrm{~h}$, and have been correlated with time of exposure, gender and SpO2, but not with NT-proBNP. Appearance of multiple BLA during exposure to altitude seems to reflect an individual's response to hypobaric hypoxia and represents a clinically relevant ultrasound sign predicting high altitude pulmonary edema (HAPE) [54]. In the cardiology setting, diffuse and multiple BLA represent a bedside ultrasound pattern of extravascular lung water, more frequently associated with left ventricular diastolic and/or systolic dysfunction. BLA can usefully integrate the clinical and pathophysiological information provided by conventional 2D and Doppler echocardiography, in patients with suspected heart failure and dyspnoea as a presenting symptom $[55,56]$. BLA resolution appears to occur in real time as fluid is removed from the body and this change was statistically significant in hemodialysis and acute decompensated heart failure. These data support thoracic ultrasound as a useful and potentially superior method for evaluating real time changes in EVLW and in assessing a patient's physiologic response to the removal of fluid [57].

Agricola et al investigated the BLA number in pulmonary edema in comparison to wedge pressure and concluded that the presence and number of BLA images provide reliable information on interstitial pulmonary edema. Therefore, bedside point-of-care ultrasonography represents an attractive, easy to use diagnostic tool for assessing both cardiac function and pulmonary congestion [58]. Monitoring pulmonary congestion is a validated technique in heart failure patients. A semi-quantification of BLA is based on a rough count of the percentage of the image occupied by the artefacts, that may be $0,50 \%$ or $100 \%$, calculated on the sum of the whole chest observation of 28 rib spaces [33].

\section{Interstitial lung diseases}

The ultrasound assessment of interstitial lung diseases (ILD) is determined by the presence and semiquantification of BLA. Hasan, et al evaluated 61 patients with ILD and assessed the presence of BLA and the distance between them. All patients had diffuse bilateral BLA [59]. The distance between each of the two adjacent BLA correlated with the severity of disease on chest HRCT, where $\mathrm{B} 3$ (the distance was $3 \mathrm{~mm}$ ) correlated with ground glass opacity and B7 (the distance was $7 \mathrm{~mm}$ ) correlated with extensive fibrosis and honeycombing. In addition, the distance between BLA inversely correlated with FVC (forced vital capacity), TLC (total lung capacity), DLCO (diffusion capacity for carbon monoxide) and $\mathrm{PaO} 2$ (partial arterial oxygen pressure). Multiple BLA seem to be useful in the first assessment of ILD. Ultrasound is able to detect early stages of pulmonary changes and subclinical lung involvement [59].

Cogliati $\mathrm{C}$ et al evaluated the accuracy of lung ultrasound in the diagnosis of ILD in patients with rheumatoid arthritis (RA) and validated the use of lung ultrasound as a screening tool to predict pulmonary involvement in the disease. The sensitivity and specificity of standard lung ultrasound vs HRCT were $92 \%$ and $56 \%$, respectively. A BLA score was significantly correlated with HRCT score $(\mathrm{r}=0.806)$. The strong correlation between echographic and HRCT scores indicates that lung ultrasound is a valid tool for grading and follow-up of ILD [28].

Patients with a diagnosed systemic connective tissue disease require regular monitoring for interstitial lung disease. Buda et al described the criteria for pulmonary fibrosis and the degree of the severity of fibrosis during the course of interstitial lung disease, through transthoracic lung ultrasound. BLA were observed in $92.3 \%$ and the authors specified three types of the observed artefacts: single BLA ( $\leq 3$ per one scan), numerous BLA ( $\geq 4$ per one scan), white lung. Single B line artefacts occurred only in the lower fields. Numerous B line artefacts were observed in $69.3 \%$ of patients from the group tested, mostly in the lower fields. They suggested a BLA cut-off value of BLA $\geq 4$ as the criteria for pulmonary fibrosis in transthoracic 
lung ultrasound [60]. The value of ultrasound in sarcoidosis has been recently summarized $[7,8,10,61]$ (fig 4).

Focal interstitial syndrome

The focal interstitial syndrome can be detected in many conditions and is especially associated with pleuritic pain. Gehmacher et al described patients with pleurisy showing a rough irregular appearance of the pleura in $89 \%$ of cases, with small subpleural consolidations in $64 \%$ and a localized effusion in 64\% [62]. The sensitivity, specificity, positive, and negative predictive values and accuracy of LUS in the diagnosis of radio-occult lesions was $94.7 \%$, $96.7 \%, 94.7 \%, 96.7 \%$, and $95.9 \%$, respectively.

Other studies showed that in patients with pleuritic pain of unknown cause, real time LUS enables the diagnosis of radio-occult lung and pleural lesions $[63,64]$. Eighteen percent of patients with lung contusion were found to have lung consolidation [65], but a focal interstitial syndrome may be in itself, a sufficiently reliable early sign of contusion [64]. A focal sonographic pattern of the interstitial syndrome may also be seen at the margin of pneumonia, in pulmonary infarction, atelectasis and neoplasia. Therefore, it is necessary to consider the focal interstitial syndrome within the context of the history and clinical findings.

\section{Pneumonia}

Meta-analyses confirm that pneumonia can be diagnosed using lung ultrasound [21,66] (fig 5). Caiulo et al described the ultrasonographic appearance of community acquired pneumonia at presentation and during the follow up of 102 patients with clinical signs and symptoms of pneumonia. BLA were often seen in the areas adjacent to the consolidation, probably as an expression of inflammatory perilesional edema. Pleural line abnormalities and pleural effusions were always associated with areas of confluent BLA and/or lung consolidation. During the follow up of more than 14 days, in all 59 areas of confluent BLA, a disappearance of BLA or a change in the pattern of multiple isolated BLA, was observed as a sign of lung reaeration. The authors concluded that ultrasound is a simple, reliable and radiation free imaging tool, not inferior to chest $\mathrm{X}$ ray, to identify pleural pulmonary alterations in children with suspected pneumonia [67]. Lung ultrasound showed a sensitivity of $96.97 \%$ and a specificity of $96.49 \%$ in predicting radio-occult pleural pulmonary lesions, and a significantly higher area under the curve (AUC) of receiver operating characteristic analysis compared with D-dimer and white blood cell count.

\section{Systemic sclerosis}

Early detection of disease manifestations like pulmonary hypertension and interstitial lung disease is of vital importance in systemic sclerosis patients. Moazedi-Fuerst et al tested the reliability of lung ultrasound for the assessment of patients with systemic sclerosis.

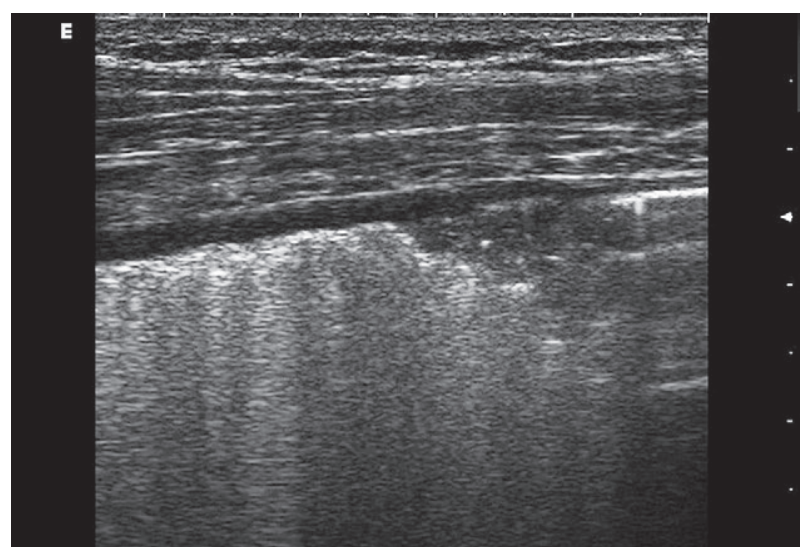

Fig 4. Sarcoidosis. Interrupted pleura line and small subpleural consolidations, typical for sarcoidosis.

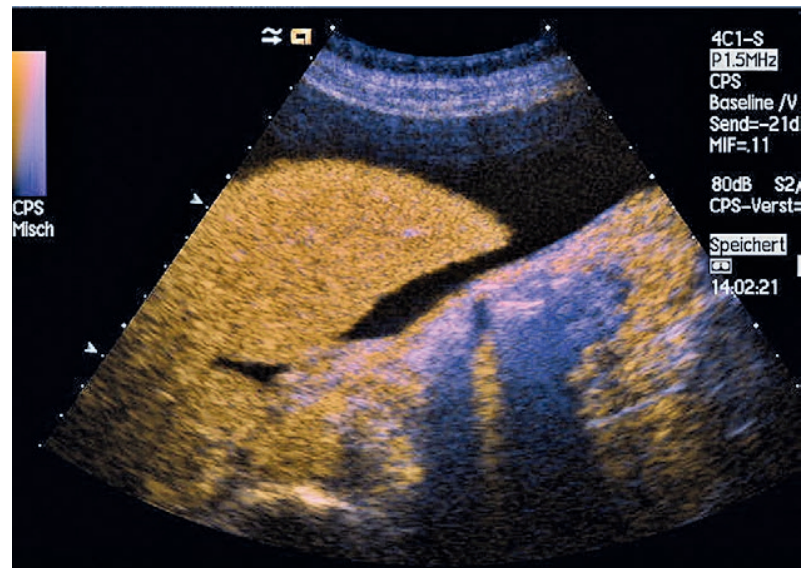

Fig 5. Pneumonia. Contrast enhanced ultrasound excludes abscess formation.

About $44 \%$ of systemic sclerosis patients showed BLA appearance and pleural irregularities. Lung ultrasound might prove a suitable method for screening patients with systemic sclerosis for incipient pulmonary structural changes [68].

Gigante et al evaluated lung ultrasound in systemic sclerosis and performed a correlation analysis with high resolution $\mathrm{CT}$, pulmonary function tests and clinical variables of disease. They found that the number of BLA increased with the progression of both high resolution CT score and digital vascular damage [69].

Chronic obstructive pulmonary disease

Lung ultrasound appears to be particularly useful in differentiating exacerbations of chronic obstructive pulmonary disease, a condition that does not show BLA, from decompensated heart failure [22].

Acute respiratory failure

The primary diagnosis of pulmonary interstitial fluid in the emergency setting is also crucial for the differentiating between cardiogenic and non-cardiogenic factors 
determining acute respiratory failure [70]. BLA have been demonstrated as a useful primary diagnostic test in acute respiratory failure patients. In patients with acute lung injury (ALI/ARDS), a given degree of lung aeration (referring to well defined CT scan entities) corresponds to a specific ultrasound pattern. Loss of aeration can be qualitatively estimated as complete (ultrasound consolidation, corresponding to $\mathrm{CT}$ consolidation), or partial (coalescent BLA, corresponding to $\mathrm{CT}$ ground glass or multiple spaced BLA corresponding to $\mathrm{CT}$ interstitial edema). Four stages of lung aeration can be evidenced using LUS; 1) Normal aeration, defined as the presence of lung sliding with horizontal A lines or non-significant few BLA; 2) moderate loss of aeration, defined as the presence of multiple vertical BLA with well-defined spacing, usually due to interstitial edema or fibrosis; 3 ) severe loss of aeration, defined as the presence of coalescent BLA less than $3 \mathrm{~mm}$ apart and resulting from partial filling of alveolar spaces by pulmonary edema or confluent bronchopneumonia; 4) complete loss of aeration, defined as the presence of consolidation $[23,32]$.

Lung ultrasound is a readily available bedside tool and the correlation of BLA with EVLW has been demonstrated in animals, extreme environments and a range of clinical settings showing good sensitivity and specificity. EVLW is also an independent risk factor of 28 day mortality in patients with ARDS [71].

Jambrik et al estimated the correlation between BLA number and the wet/dry ratio of the lung tissue measured by gravimetry, in an experimental pig model of ALI/ ARDS. They concluded that BLA assessed by lung ultrasound provide a simple, semi-quantitative, noninvasive index of lung water accumulation, strongly correlated to invasive gravimetric assessment. BLA could quantify lung edema noninvasively in real time. BLA detected by lung ultrasound were proposed as a useful, practical and appealing simple way to image directly extravascular lung water (EVLW) [72].

Bataille et al assessed the correlation and the trending ability between EVLW measured by transpulmonary thermodilution and the BLA score or the E/Ea ratio in patients with ARDS. They concluded that the correlation between EVLW changes and BLA variations was significant. BLA score $\geq 6$ had a sensitivity of $82 \%$ and a specificity of $77 \%$ to predict EVLW $>10 \mathrm{ml} / \mathrm{kg}$. The correlation between ultrasound BLA and EVLW was significant, but the BLA score was not able to track EVLW changes reliably [26].

Monitoring (e.g., ventilated patients)

Enghard et al evaluated a four-region protocol of lung ultrasound to determine the pulmonary fluid status in ventilated patients in the intensive care unit. Lung wa- ter assessment by ultrasound using a simplified protocol showed excellent correlation with extravascular lung water index (EVLWI) over a broad range of lung hydration grades and ventilator settings. A US score $>1.5$ had a sensitivity and specificity of $92.1 \%$ and $91.7 \%$ for diagnosing an EVLWI above the normal value of $7 \mathrm{ml} / \mathrm{kg}$. To identify patients with a severely increased EVLWI $>15$, a US score of $>18.5$ had a sensitivity of $92.3 \%$ and specificity of $94.6 \%$. Thus, lung ultrasound is a useful, non-invasive tool in predicting hydration status in mechanically ventilated patients. The four region protocol is time saving, correlates well with transpulmonary thermodilution measurements and performs markedly better than chest radiography [73].

Monitoring of different states, e.g., fluid overload in hemodialysis, semi-quantification of extravascular lung water and pulmonary aeration, has been studied.

The change in BLA is rapid and BLA respond quickly to changes in lung water content. Thus, as a fluid overloaded patient is dialyzed, it is possible to track BLA resolution in real time as fluid, and therefore extravascular lung water, is removed from the body [38,57]. A significant correlation was found between the number of BLA and extra vascular water in maintenance haemodialysis (MHD) patients. The dynamic changes in BLA after hemodialysis are correlated to the changes in total body and extra vascular water, and particularly to lung fluid removal. B line assessment in MHD patients is highly feasible with a simplified and timely scanning scheme limited to the lateral chest regions. These premises make BLA a promising biomarker for a bedside assessment of pulmonary congestion in MHD patients [74].

As a monitoring tool in a prehospital emergency setting, findings from lung ultrasound scans correspond with improved hemodynamic parameters in patients with acute decompensated heart failure (ADHF) treated with continuous positive airway pressure (CPAP) compared with standard therapy only [75]. The number of sonographic BLA correlated well with the radiologic estimate of extravascular lung water. In patients with cardiogenic pulmonary edema, evaluation of BLA and a change (decrease) in their number enables a noninvasive monitoring of response to therapy [24]. BLA are also considered a sign of loss of aeration in critically ill patients admitted to a polyvalent ICU, and monitoring change of aeration in time is a useful technique to orientate recruitment maneuvers and ventilator strategies [76].

\section{A line artefacts (ALA)}

ALA are defined as repetitive horizontal artefacts arising from the pleural line. They are generated by subpleural air, which blocks the propagation of the ultrasound beam. The normal lung surface associates lung sliding with horizontal repetitions of the pleural line, in 
a phenomenon of repetitive mirroring of the image of the chest wall below the pleural line. A-lines indicate gas (physiological or free). Lung sliding is a to-and-fro movement at the pleural line, synchronous with the respiratory movement. A lines are not always clearly visible, because their visualization depends on the interface, the probe orientation and angle, the frequency and probe used. A-lines may typically be more clearly visible in a condition of free air (pneumothorax) [21], but also in a condition of increased pulmonary air content (severe emphysema or alveolar overdistension in excess of ventilation). However, opposite to BLA, A-lines have no pathologic meaning. For this reason, even without a clear visualization of A-lines, it is decisive a pattern with no lung sliding and no BLA to identify a pneumothorax [77]. Also, in a condition of lung sliding without BLA, even in the absence of clearly visible A-lines, it is possible to rule out an interstitial syndrome. Thus, in practice it is better to speak about an A-profile (absence of BLA) with or without sliding, rather than giving importance to the visualization of A-lines. Of course, in the absence of clearly visible A-lines, the white lung (multiple merging BLA described also in patients with acute respiratory distress syndrome) and a consolidated lung, should be excluded and differentiated from the A-profile.

\section{Open questions}

Open questions include the ease with which lung ultrasound utilization will pass from early adopters and point of care enthusiasts to the ordinary provider. Early indications are that like ultrasound visionaries, ordinary clinicians are adopting lung ultrasound at a rapid pace due to its accuracy, ease of use and safety.

Another open question is the effect of protocol driven lung ultrasound use on future expansion and accuracy. Multiple protocols currently exist in lung ultrasound and it remains to be seen if a set of unified scanning protocols can be adopted for the various specialties utilizing ultrasound. Lung artefacts in contrast enhanced endobronchial ultrasound [78] and endobronchial ultrasound elastography have to be encountered [79].

\section{Conclusion}

Lung ultrasound, while an innovate application to novice providers, is a well-researched and supported ultrasound subspecialty. Understanding the various ultrasound signs and artifacts encountered in lung ultrasound examinations is critical to correct diagnosis of normal and pathologic states. Clinicians should use the findings discussed within this manuscript as a guide for their own practice and build experience.

\section{References}

1. Volpicelli G, Elbarbary M, Blaivas M, et al. International evidence-based recommendations for point-of-care lung ultrasound. Intensive Care Med 2012;38:577-591.

2. Dietrich CF, Mathis G, Blaivas M, et al. Lung B-line artefacts and their use. J Thorac Dis 2016;8:1356-1365.

3. Wastl D, Borgmann T, Helwig K, Dietrich CF. Rapid diagnostic in the emergency unit: bedside sonography. Dtsch Med Wochenschr 2016;141:317-321.

4. Wastl D, Helwig K, Dietrich CF. Examination concepts and procedures in emergency ultrasonography. Med Klin Intensivmed Notfmed 2015;110:231-239.

5. Dietrich CF, Gebhard Mathis G, Cui XW, Ignee A, Hocke $\mathrm{M}$, Hirche TM. Ultrasound of the pleurae and lungs. Ultrasound Med Biol 2015;41:351-365.

6. Dietrich CF, Hirche TO, Schreiber D, Wagner TO. Sonographie von Pleura und Lunge. Ultraschall Med 2003;24:303311.

7. Dietrich CF, Annema JT, Clementsen P, Cui XW, Borst $\mathrm{MM}$, Jenssen C. Ultrasound techniques in the evaluation of the mediastinum, part I: endoscopic ultrasound (EUS), endobronchial ultrasound (EBUS) and transcutaneous mediastinal ultrasound (TMUS), introduction into ultrasound techniques. J Thorac Dis 2015;7:E311-E325.

8. Jenssen C, Annema JT, Clementsen P, Cui XW, Borst MM, Dietrich CF. Ultrasound techniques in the evaluation of the mediastinum, part 2: mediastinal lymph node anatomy and diagnostic reach of ultrasound techniques, clinical work up of neoplastic and inflammatory mediastinal lymphadenopathy using ultrasound techniques and how to learn mediastinal endosonography. J Thorac Dis 2015;7:E439-E458.

9. Jenssen C, Annema, JT, Zels K, Dietrich, CF. Endosonographische Evaluierung von Lunge und Mediastinum. EndHeu 2014;27:37-44.

10. Hirche TO, Hirche H, Cui XW, Wagner TO, Dietrich CF. Ultrasound evaluation of mediastinal lymphadenopathy in patients with sarcoidosis. Med Ultrason 2014;16:194-200.

11. Tuma J, Jenssen C, Moller K, et al. Ultrasound artifacts and their diagnostic significance in internal medicine and gastroenterology - Part 1: B-mode artifacts. Z Gastroenterol 2016;54:433-450.

12. Dietrich CF, Ignee A, Greis C, Cui XW, Schreiber-Dietrich DG, Hocke M. Artifacts and pitfalls in contrast-enhanced ultrasound of the liver. Ultraschall Med 2014;35:108-125.

13. Dietrich CF, Ignee A, Hocke M, Schreiber-Dietrich D, Greis C. Pitfalls and artefacts using contrast enhanced ultrasound. Z Gastroenterol 2011;49:350-356.

14. Cui XW, Ignee A, Hocke M, Seitz K, Schrade G, Dietrich $\mathrm{CF}$. Prolonged heterogeneous liver enhancement on contrast-enhanced ultrasound. Ultraschall Med 2014;35:246252.

15. Cui XW, Pirri C, Ignee A, et al. Measurement of shear wave velocity using acoustic radiation force impulse imaging is not hampered by previous use of ultrasound contrast agents. Z Gastroenterol 2014;52:649-653. 
16. Bonhof JA. Ultrasound Artifacts - Part 1. Ultraschall Med 2016;37:140-153.

17. Avruch L, Cooperberg PL. The ring-down artifact. J Ultrasound Med 1985;4:21-28.

18. Bessis L, Callard P, Gotheil C, Biaggi A, Grenier P. Highresolution $\mathrm{CT}$ of parenchymal lung disease: precise correlation with histologic findings. Radiographics 1992;12:45-58.

19. Webb WR. Thin-section CT of the secondary pulmonary lobule: anatomy and the image--the 2004 Fleischner lecture. Radiology 2006;239:322-338.

20. Soldati G, Smargiassi A, Inchingolo R, et al. Lung ultrasonography may provide an indirect estimation of lung porosity and airspace geometry. Respiration 2014;88:458468.

21. Volpicelli G. Lung sonography. J Ultrasound Med 2013;32:165-171.

22. Volpicelli G, Cardinale L, Garofalo G, Veltri A. Usefulness of lung ultrasound in the bedside distinction between pulmonary edema and exacerbation of COPD. Emerg Radiol 2008;15:145-151.

23. Bouhemad B, Liu ZH, Arbelot C, et al. Ultrasound assessment of antibiotic-induced pulmonary reaeration in ventilator-associated pneumonia. Crit Care Med 2010;38:84-92.

24. Volpicelli G, Mussa A, Garofalo G, et al. Bedside lung ultrasound in the assessment of alveolar-interstitial syndrome. Am J Emerg Med 2006;24:689-696.

25. Chiesa AM, Ciccarese F, Gardelli G, et al. Sonography of the normal lung: Comparison between young and elderly subjects. J Clin Ultrasound 2014 Sep 15. doi: 10.1002/ jcu. 22225 .

26. Bataille B, Rao G, Cocquet P, et al. Accuracy of ultrasound $\mathrm{B}$-lines score and $\mathrm{E} / \mathrm{Ea}$ ratio to estimate extravascular lung water and its variations in patients with acute respiratory distress syndrome. J Clin Monit Comput 2015;29:169-176.

27. Brattain LJ, Telfer BA, Liteplo AS, Noble VE. Automated B-Line Scoring on Thoracic Sonography. J Ultrasound Med 2013;32:2185-2190.

28. Cogliati C, Antivalle M, Torzillo D, et al. Standard and pocket-size lung ultrasound devices can detect interstitial lung disease in rheumatoid arthritis patients. Rheumatology (Oxford) 2014;53:1497-1503.

29. Sperandeo M, Varriale A, Sperandeo G, et al. Assessment of ultrasound acoustic artifacts in patients with acute dyspnea: a multicenter study. Acta Radiologica 2012;53:885-892.

30. Reissig A, Kroegel C. Transthoracic sonography of diffuse parenchymal lung disease: the role of comet tail artifacts. J Ultrasound Med 2003;22:173-180.

31. Agricola E, Bove T, Oppizzi M, et al. "Ultrasound comettail images": a marker of pulmonary edema: a comparative study with wedge pressure and extravascular lung water. Chest 2005;127:1690-1695.

32. Lichtenstein D, Meziere G, Biderman P, Gepner A, Barre O. The comet-tail artifact. An ultrasound sign of alveolar-interstitial syndrome. Am J Respir Crit Care Med 1997; 156:1640-1646.

33. Gargani L. Lung ultrasound: a new tool for the cardiologist. Cardiovasc Ultrasound 2011;9:6.
34. Boussuges A, Coulange M, Bessereau J, et al. Ultrasound lung comets induced by repeated breath-hold diving, a study in underwater fishermen. Scand J Med Sci Sports 2011;21:e384-e392.

35. Fagenholz PJ, Gutman JA, Murray AF, Noble VE, Thomas SH, Harris NS. Chest ultrasonography for the diagnosis and monitoring of high-altitude pulmonary edema. Chest 2007;131:1013-1018.

36. Jambrik Z, Monti S, Coppola V, et al. Usefulness of ultrasound lung comets as a nonradiologic sign of extravascular lung water. Am J Cardiol 2004;93:1265-1270.

37. Liteplo AS, Marill KA, Villen T, et al. Emergency thoracic ultrasound in the differentiation of the etiology of shortness of breath (ETUDES): sonographic B-lines and N-terminal pro-brain-type natriuretic peptide in diagnosing congestive heart failure. Acad Emerg Med 2009;16:201-210.

38. Mallamaci F, Benedetto FA, Tripepi R, et al. Detection of pulmonary congestion by chest ultrasound in dialysis patients. JACC Cardiovasc Imaging 2010;3:586-594.

39. Pivetta E, Goffi A, Lupia E, et al. Lung ultrasound-implemented diagnosis of acute decompensated heart failure in the ED: A SIMEU multicenter study. Chest 2015;148:202210.

40. Anderson KL, Fields JM, Panebianco NL, Jenq KY, Marin I, Dean AJ. Inter-rater reliability of quantifying pleural blines using multiple counting methods. J Ultrasound Med 2013;32:115-120.

41. Sperandeo M, Carnevale V, Muscarella S, et al. Clinical application of transthoracic ultrasonography in inpatients with pneumonia. Eur J Clin Invest 2014;41:1-7.

42. Gullett J, Donnelly JP, Sinert R, et al. Interobserver agreement in the evaluation of $\mathrm{B}$ lines using bedside ultrasound. J Crit Care 2015;30:1395-1399.

43. Ciccarese F, Chiesa AM, Feletti F, et al. The senile lung as a possible source of pitfalls on chest ultrasonography and computed tomography. Respiration 2015;90:56-62.

44. Chavez MA, Naithani N, Gilman RH, et al. Agreement between the world health organization algorithm and lung consolidation identified using point-of-care ultrasound for the diagnosis of childhood pneumonia by general practitioners. Lung 2015;193:531-538.

45. Martelius L, Heldt H, Lauerma K. B lines on Pediatric Lung Sonography: Comparison With Computed Tomography. J Ultrasound Med 2016;35:153-157.

46. Gargani L, Volpicelli G. How I do it: Lung ultrasound. Cardiovasc Ultrasound 2014;12:25.

47. Facchini C, Malfatto G, Giglio A, Facchini M, Parati G, Branzi G. Lung ultrasound and transthoracic impedance for noninvasive evaluation of pulmonary congestion in heart failure. J Cardiovasc Med (Hagerstown) 2016;17:510-517.

48. Miglioranza MH, Gargani L, Sant'Anna RT, et al. Lung ultrasound for the evaluation of pulmonary congestion in outpatients: a comparison with clinical as-sessment, natriuretic peptides, and echocardiography. JACC Cardiovasc Imaging 2013;6:1141-1151.

49. Weber CK, Miglioranza MH, Moraes MA, et al. The fivepoint Likert scale for dyspnea can properly assess the de- 
gree of pulmonary congestion and predict adverse events in heart failure outpatients. Clinics (Sao Paulo) 2014;69:341346.

50. Frassi F, Pingitore A, Cialoni D, Picano E. Chest sonography detects lung water accumulation in healthy elite apnea divers. J Am Soc Echocardiogr 2008;21:1150-1155.

51. Edsell ME, Wimalasena YH, Malein WL, et al. High-intensity intermittent exercise increases pulmonary interstitial edema at altitude but not at simulated altitude. Wilderness Environ Med 2014;25:409-415.

52. Volpicelli G, Skurzak S, Boero E, et al. Lung ultrasound predicts well extravascular lung water but is of limited usefulness in the prediction of wedge pressure. Anesthesiology 2014; 121:320-327.

53. Gargani L, Frassi F, Soldati G, Tesorio P, Gheorghiade M, Picano E. Ultrasound lung comets for the differential diagnosis of acute cardiogenic dyspnoea: A comparison with natriuretic peptides. Eur JHeart Fail 2008;10:70-77.

54. Strapazzon G, Vezzaro R, Hofer G, et al. Factors associated with B-lines after exposure to hypobaric hypoxia. Eur Heart J Cardiovasc Imaging 2015;16:1241-1246.

55. Frassi F, Gargani L, Gligorova S, Ciampi Q, Mottola G, Picano E. Clinical and echocardiographic determinants of ultrasound lung comets. Eur J Echocardiogr 2007;8:474479.

56. Frassi F, Gargani L, Tesorio P, Raciti M, Mottola G, Picano E. Diagnostic value of extravascular lung water assessed with ultrasound lung comets by chest sonography in patients with dyspnea and/or chest pain. J Card Fail 2007; 13:830-835.

57. Noble VE, Murray AF, Capp R, Sylvia-Reardon MH, Steele DJ, Liteplo A. Ultrasound assessment for extravascular lung water in patients undergoing hemodialysis. Chest 2009;135:1433-1439.

58. Agricola E, Bove T, Oppizzi M, et al. "Ultrasound comettail images": a marker of pulmonary edema: a comparative study with wedge pressure and extravascular lung water. Chest 2005;127:1690-1695.

59. Hasan AA, Makhlouf HA. B-lines: Transthoracic chest ultrasound signs useful in assessment of interstitial lung diseases. Ann Thorac Med 2014;9:99-103.

60. Buda N, Piskunowicz M, Porzezińska M, Kosiak W, Zdrojewski Z. Lung Ultrasonography in the Evaluation of Interstitial Lung Disease in Systemic Connective Tissue Diseases: Criteria and Severity of Pulmonary Fibrosis - Analysis of 52 Patients. Ultraschall Med 2016;37:379-385.

61. Tana C, Silingardi M, Dietrich CF. New trends in ultrasound of hepatosplenic sarcoidosis. Z Gastroenterol 2015;53:283284.

62. Gehmacher O, Kopf A, Scheier M, Bitschnau R, Wertgen T, Mathis G. Can pleuricy be detected with ultrasound? Ultraschall Med 1997;18:214-219.

63. Volpicelli G, Caramello V, Cardinale L, Cravino M. Diagnosis of radio-occult pulmonary conditions by realtime chest ultrasonography in patients with pleuritic pain. Ultrasound Med Biol 2008;34:1717-1723.
64. Soldati S, Testa A, Silva FR, Carbone L, Portale G, Silveri NG. Chest ultrasonography in lung contusion. Chest 2006; 130:533-538.

65. Wüstner A, Gehmacher O, Hämmerle S, Schenkenbach C, Häfele H, Mathis G. Ultrasound diagnosis in blunt thoracic trauma. Ultraschall Med 2005;26:285-290.

66. Blaivas M. Lung ultrasound in evaluation of pneumonia. J Ultrasound Med 2012;31:823-826.

67. Caiulo VA, Gargani L, Caiulo S, et al. Lung ultrasound characteristics of community-acquired pneumonia in hospitalized children. Pediatr Pulmonol 2013;48:280-287.

68. Moazedi-Fuerst FC, Kielhauser S, Brickmann K, et al. Sonographic assessment of interstitial lung disease in patients with rheumatoid arthritis, systemic sclerosis and systemic lupus erythematosus. Clin Exp Rheumatol 2015;33:S87-S91.

69. Gigante A, Rossi Fanelli F, Lucci S, et al. Lung ultrasound in systemic sclerosis: correlation with high-resolution computed tomography, pulmonary function tests and clinical variables of disease. Intern Emerg Med 2016;11:213-217.

70. Copetti R, Soldati G, Copetti P. Chest sonography: a useful tool to differentiate acute cardiogenic pulmonary edema from acute respiratory distress syndrome. Cardiovasc U1trasound 2008;6:16.

71. Shyamsundar M, Attwood B, Keating L, Walden AP. Clinical review: the role of ultrasound in estimating extra-vascular lung water. Crit Care 2013;17:237.

72. Jambrik Z, Gargani L, Adamicza A, et al. B-lines quantify the lung water content: a lung ultrasound versus lung gravimetry study in acute lung injury. Ultrasound Med Biol 2010;36:2004-2010.

73. Enghard P, Rademacher S, Nee J, et al. Simplified lung ultrasound protocol shows excellent prediction of extravascular lung water in ventilated in-tensive care patients. Critical Care 2015;19:36.

74. Donadio C, Bozzoli L, Colombini E, et al. Effective and timely evaluation of pulmonary congestion: qualitative comparison between lung ultrasound and thoracic bioelectrical impedance in maintenance hemodialysis patients. Medicine (Baltimore) 2015;94:e473.

75. Strnad M, Prosen G, Borovnik Lesjak V. Bedside lung ultrasound for monitoring the effectiveness of prehospital treatment with continuous positive airway pressure in acute decompensated heart failure. Eur J Emerg Med 2016;23:5055.

76. Bouhemad B, Brisson H, Le-Guen M, Arbelot C, Lu Q, Rouby JJ. Bedside ultrasound assessment of positive endexpiratory pressure-induced lung recruitment. Am J Respir Crit Care Med 2011;183:341-347.

77. Piette E, Daoust R, Denault A. Basic concepts in the use of thoracic and lung ultrasound. Curr Opin Anesthesiol 2013;26:20-30.

78. Dietrich CF. Contrast Enhanced EndoBronchial UltraSound (CE-EBUS), potential value of a new method. Endosc U1trasound 2016, in press.

79. Dietrich CF, Jenssen C, Herth FJ. Endobronchial ultrasound elastography. Endosc Ultrasound 2016;5:233-238. 